\title{
Infraestruturas Críticas no Processo de Graduação em Instituições Federais de Ensino Superior do Ceará
}

\author{
Massaro Victor Pinheiro Alves ${ }^{1}$, Bruno Correia da Silva ${ }^{1}$, Adriano de Oliveira \\ Maia $^{1}$, Rangel Henrique Félix ${ }^{1}$ \\ ${ }^{1}$ Instituto Federal de Educação, Ciência e Tecnologia do Ceará (IFCE) \\ Rua Pedro Bezerra de Menezes, 387, COHAB - 63.475-000 - Jaguaribe - CE - Brasil \\ victormassaro00@gmail.com, bruno.silva@ifce.edu.br, \\ adhryan.olivirmayer@gmail.com, rangel.crede11@gmail.com
}

\begin{abstract}
This article analyzes the Pedagogical Projects of the Undergraduate Courses (PPUC) of the Federal University of Ceará and the Federal Institute of Ceara aiming to verify if the institutions treat the domains of Critical Infrastructures during the Undergraduate Program. The method of collecting the material was carried out through the institutions' website with the analysis process based on descriptors found on the National Guide for the Safety of CIs. It was observed in the results a tenuous and indirect association from the CIs in relation to the contents of the PPUCs, and finally, it was made a reflexion on the importance of them during the training of professionals who will deal with such responsibilities.
\end{abstract}

Resumo. Este artigo analisa os Projetos Pedagógicos dos Cursos (PPC) de cursos da Universidade Federal do Ceará e do Instituto Federal do Ceará, com a pretensão de verificar se as instituições tratam o tema Infraestruturas Críticas (IC) durante a graduação. $O$ método de coleta do material se operacionalizou através de consulta aos sites das instituições, com o processo de análise baseado em descritores fundamentados no Guia Nacional para a Segurança das IC. Observou-se nos resultados uma associação tênue e indireta destas quanto aos conteúdos dos PPC. Por fim, faz-se uma reflexão acerca da importância de estes serem abordados de forma ampla, durante a formação dos profissionais que lidarão com tais responsabilidades.

\section{Introdução}

As Infraestruturas Críticas (IC) são instalações, serviços, bens e sistemas que, se forem interrompidos ou destruídos, provocarão impacto nos âmbitos social, econômico e político, afetando as relações internacionais e a Segurança Nacional [Canongia et al. 2010]. A Infraestruturas que mantém o Facebook e o WhatsApp é um exemplo comercial, já a rede elétrica brasileira é um serviço estatal de serviço. Os desastres naturais (tempestades, furacões, terremotos, etc); catástrofes provocadas pelo homem (terrorismo, ataques físicos, ataques cibernéticos); e outros fatores (por exemplo, epidemias e interrupções elétricas) [Mishra et al. 2015] podem comprometer uma IC, mas são fatores que podem ser previsíveis para se atuar na manutenção. Este artigo propõe-se a evidenciar a falta de relevância que é observada no ensino, no caso, o ensino 
superior, após o qual o profissional estaria apto a utilizar essas infrasestruturas, mas tem sua habilidade comprometida/não desenvolvida por falta de conhecimento.

De acordo com Hosang (2011), num país de dimensões continentais, como o Brasil, as IC possuem caráter estratégico, desempenhando um papel essencial, tanto para a segurança e soberania nacional quanto para a integração cultural e o desenvolvimento econômico, e são por essas razões um objetivo estratégico e permanente.

Com base nesses conceitos, reflete-se sobre a necessidade de se conscientizar a comunidade da relevância e forte ligação das IC com o bem-estar social, buscando-se propagar o conhecimento acerca destas. Partindo disso, decidiu-se verificar como as instituições federais do Ceará tratam o assunto, analisando-se os Projetos Pedagógicos dos Cursos (PPC) de graduação da Universidade Federal do Ceará (UFC) e do Instituto Federal do Ceará (IFCE).

\section{Fundamentação Teórica}

O Departamento de Segurança da Informação e Comunicações do Brasil publicou em 2010 o Guia de Referência para a Segurança das Infraestruturas Críticas da Informação. Entre os argumentos, é ressaltada a importância da elaboração de diretrizes, planos e ações voltados a assegurar e promover a segurança das IC, tendo em vista a transversalidade e particularidade do assunto [Canongia et al. 2010].

Lewis (2014), enfatiza que as Infraestruturas Críticas possuem características variadas e peculiares, fazem-se constantes em consideráveis disciplinas e jurisdições, transitando nos âmbitos federal, estadual e municipal, estando presente em organizações públicas e privadas. É necessário observar que manejar IC pode acarretar lidar com questões políticas em um extremo e, no outro, com um diversificado conjunto científico.

Considerando os fatores expostos, chegamos à premissa da relevância de se formarem profissionais capacitados e com conhecimento prévio sobre as IC. Mishra et al (2013) são enfáticos sobre a importância de a universidade prover, ao longo da trajetória educacional, habilidades e conhecimentos necessários para abordar a resiliência e o tratamento de situações adversas em IC.

A partir de artigo de Matos (2012), em que apresenta uma reflexão sobre o perfil do discente e sua coerência com a prática social desse indivíduo formado, cruza-se a análise com a pauta das IC. Nesse sentido, este estudo propõe identificar se esse profissional, na região cearense, no ensino superior público federal, tem na sua formação similiraridades ou disparidades nos PPC dos cursos.

\section{Metodologia}

O artigo se estabeleceu com a análise dos Projetos Pedagógicos dos Cursos (PPC) de graduações da Universidade Federal do Ceará (UFC) e do Instituto Federal do Ceará (IFCE), verificando se ambos abordam as IC durante essa formação. A escolha dessas instituições deu-se por sua relevância no estado, uma vez que formam profissionais qualificados e valorizados para a sociedade.

Quanto à abordagem, trata-se de uma pesquisa qualitativa que se desenha com a análise documental dos PPC. O processo de busca dos documentos iniciou-se com a coleta do material nos sites das duas instituições. Em seguida, a análise começou com a 
definição dos descritores (palavras-chave que auxiliarão a procura). Estes foram ordenados em temas, que estão presentes na Tabela 1, e descritos abaixo, fundamentados no Guia Nacional de Referência para a Segurança das IC da Informação [Canongia et al. 2010]. Sintetizaram-se as referências, expuseram-se as motivações e determinaram-se as bases da pesquisa, subdividindo-se os descritores em grupos, visando maximizar a abrangência do assunto. Essa distribuição teve o intuito de potencializar as buscas, com vistas a englobar toda a temática das IC, para que alguns fatores não ficassem ausentes durante o processo.

O Tema I, Infraestruturas Críticas, é o fator fundamental e central da pesquisa. Para aprimorar os resultados, estabeleceu-se uma divisão das IC em subcategorias, que englobam fundamentalmente a mesma ideia, decompondo-se o assunto em descritores complementares: Sistemas Críticos e Sistemas de Infraestruturas Críticas.

O Tema II procurou compor e ampliar a busca, focando em uma área que complementa as IC, a Gestão de Riscos, sendo que as organizações estão expostas a fatores internos e externos, e se utilizam disso para analisar e prevenir os riscos, a fim de atenderem os critérios e requisitos necessários à continuidade de suas operações.

No Tema III, englobaram-se mais fatores, chegando-se à contextualização com a parte tecnológica e abordando-se a premissa do papel das IC como uma organização que compreende a área da Gestão da Informação, provendo requisitos de segurança e controles para proteção e gestão de sistemas e processos de forma sistemática.

No Tema IV, incluiu-se a relação das IC na conjuntura da área da Segurança da Informação, seguindo-se princípios e processos que devem ser utilizados para garantir e minimizar os perigos, com foco no nível operacional da segurança, bem como na proteção dos ativos e tratamento dos riscos associados à Segurança Cibernética.

Tabela 1. Maximização dos descritores no processo de busca

\begin{tabular}{|c|c|c|}
\hline & Tema & Descritores \\
\hline \multirow{3}{*}{$\mathrm{I}$} & \multirow{3}{*}{ Infraestruturas Críticas } & Sistemas Críticos \\
\hline & & Infraestruturas Críticas \\
\hline & & Sistemas de Infraestruturas Críticas \\
\hline \multirow{3}{*}{ II } & \multirow{3}{*}{ Gestão de Riscos } & Gestão de Riscos \\
\hline & & Análise de Riscos \\
\hline & & Prevenção de Riscos \\
\hline \multirow{3}{*}{ III } & \multirow{3}{*}{ Gestão da Informação } & Gestão da Informação \\
\hline & & Gestão de Processos \\
\hline & & Gestão de Sistemas \\
\hline \multirow{3}{*}{ IV } & \multirow{3}{*}{ Segurança da Informação } & Segurança da Informação \\
\hline & & Proteção de Ativos \\
\hline & & Segurança Cibernética \\
\hline
\end{tabular}


Avançando-se com a análise dos PPC, depois de se coletar o material, procedeuse a verificação da presença dos descritores nos documentos, com o objetivo de se explorarem os temas relatados na Tabela 1.

\section{Resultados}

Os PPC, depois de passarem pela análise dos descritores, foram ordenados em tabelas, para melhor explicar a distribuição e a concepção das buscas. As categorias foram divididas em subtópicos, para potencializar a sistematização do conteúdo, com o propósito de repassar as bases encontradas de maneira reta e objetiva. A seguir, explicam-se os resultados, descrevendo-se o contexto em que os descritores foram descobertos em cada documento.

\subsection{Tema I - Infraestruturas Críticas (Descritor Sistemas Críticos)}

É importante ressaltar que, neste tópico, apenas o descritor Sistemas Críticos obteve resoluções de buscas; os demais não foram encontrados em nenhum curso de ambas as instituições. Na UFC, campus Sobral, no curso de Engenharia da Computação, o descritor encontra-se na abordagem quanto ao acesso não autorizado a recursos computacionais, riscos da computação e sistemas críticos com relação à segurança.

No IFCE, o descritor está atribuído ao Curso de Sistemas de Informação, na disciplina de Engenharia de Software I, no desenvolvimento de sistemas críticos.

Tabelas 2. Descritor Sistemas Críticos nos cursos da UFC e do IFCE

\begin{tabular}{c|c|c}
\hline Instituição & Curso & Cidade \\
\hline UFC & Engenharia da Computação & Sobral \\
\hline IFCE & Sistemas de Informação & Crato \\
\hline
\end{tabular}

\subsection{Tema II - Gestão De Riscos}

\subsubsection{Descritor Gestão de Riscos}

O descritor Gestão de Riscos está presente na UFC, no curso de Finanças, campus Sobral, no componente curricular de Finanças Corporativas, designando a Gestão de Riscos: quanto ao Mercado a termo e futuro, entre outros.

No IFCE, está no curso de Gestão de Turismo, na disciplina de Gestão Financeira, como aspecto processual da gestão financeira.

Tabela 3. Descritor Gestão de Riscos nos cursos da UFC e do IFCE

\begin{tabular}{c|c|c}
\hline Instituiçãa & Curso & Cidade \\
\hline UFC & Finanças & Sobral \\
\hline IFCE & Gestão de Turismo & Canindé \\
\hline
\end{tabular}

\subsubsection{Descritor Análise de Riscos}

$\mathrm{Na}$ UFC, descritor está no curso de Ciências Econômicas, campus Fortaleza, nas disciplinas de Finanças Corporativas e Teoria das Decisões, designando a análise de 
riscos relativos as suas determinadas disciplinas. Já no curso de Engenharia Ambiental, nas cidades Fortaleza e Crateús, encontra-se na disciplina optativa de Análise de Riscos Ambientais, com os conceitos de riscos, objetivos e etapas da análise de riscos. No curso de Engenharia Metalúrgica, está nos objetivos da disciplina de Problemas Ambientais na Indústria Mínero-Metalúrgica.

O descritor Análise de Riscos encontra-se no IFCE, no curso de Ciências da Computação, campus Aracati, na disciplina optativa de Segurança da Informação, ressaltando a importância de ser feita a análise de riscos em sistemas de informação. Já em Matemática, campus Cedro, e em Mecatrônica Industrial, campus Sobral, é citado na disciplina de Projeto Social e Projeto Social e Educação Ambiental, advertindo sobre a necessidade de se realizar a análise dos riscos envolvidos. No curso de Engenharia Ambiental, campus Juazeiro do Norte, o descritor é destacado nas competências e habilidades para a realização da análise de riscos e/ou vulnerabilidades ambientais. Sobre o curso de Engenharia Civil, campus Morada Nova, a citação ocorre na disciplina de Economia Aplicada à Engenharia Civil, no programa de Matemática Financeira e Engenharia Econômica II, como aprendizagem da análise de riscos, incertezas, sensibilidade e seguros. No curso de Telemática, Tauá, há ocorrência em duas disciplinas: Segurança Da Informação, em que são atribuídos os princípios em segurança da informação e análise de riscos, e Gerenciamento de Projetos, com objetivo de fornecer bases de formação para um projeto, análise de riscos e custos.

Tabela 4. Descritor Análise de Riscos nos cursos da UFC e do IFCE

\begin{tabular}{c|c|c}
\hline Instituição & Curso & Cidade \\
\hline \multirow{4}{*}{ UFC } & Ciências Econômicas & Fortaleza \\
\cline { 2 - 3 } & Engenharia Ambiental & Crateús \\
\cline { 2 - 3 } & Engenharia Ambiental & Fortaleza \\
\cline { 2 - 3 } & Engenharia Metalúrgica & Fortaleza \\
\hline \multirow{4}{*}{ IFCE } & Ciência da Computação & Aracati \\
\cline { 2 - 3 } & Matemática & Cedro \\
\cline { 2 - 3 } & Mecatrônica Industrial & Sobral \\
\cline { 2 - 3 } & Engenharia Ambiental & Juazeiro do Norte \\
\cline { 2 - 3 } & Engenharia Civil & Morada Nova \\
\cline { 2 - 3 } & Telemática & Tauá \\
\hline
\end{tabular}

\subsubsection{Descritor Prevenção de Riscos}

O descritor Prevenção de Riscos está presente na UFC, apenas no curso de Geologia, entre competências profissionais, com o intuito de promover a prevenção de riscos de acidentes por desastres naturais e aqueles provocados pelo homem.

No IFCE, o descritor está presente nos cursos de Engenharia de Aquicultura, em Aracati, na disciplina de Gestão e Educação Ambiental, com o objetivo relatado em sua ementa de conhecer as medidas de controle de prevenção de riscos ambientais. Todos os 
VI Congresso Brasileiro de Informática na Educação (CBIE 2017)

Anais do XXIII Workshop de Informática na Escola (WIE 2017)

demais cursos mencionados na Tabela 5 apresentam de forma similar a adoção do componente curricular de Higiene e Segurança do Trabalho, tendo como foco e característica o programa de prevenção de riscos ambientais.

Tabelas 5. Descritor Prevenção de Riscos nos cursos da UFC e do IFCE

\begin{tabular}{c|c|c}
\hline Instituição & Curso & Cidade \\
\hline \multirow{2}{*}{ UFC } & Geologia & Fortaleza \\
\hline \multirow{4}{*}{ IFCE } & Engenharia de Aquicultura & Aracati \\
\cline { 2 - 3 } & Engenharia de Aquicultura & Morada Nova \\
\cline { 2 - 3 } & Automação Industrial & Juazeiro do Norte \\
\cline { 2 - 3 } & Engenharia de Controle e Automação & Maracanaú \\
\cline { 2 - 3 } & Tecnologia em Alimentos & Sobral \\
\cline { 2 - 3 } & Saneamento Ambiental & Sobral \\
\cline { 2 - 3 } & Irrigação e Drenagem & Sobral \\
\hline
\end{tabular}

\subsection{Tema III - Gestão da Informação}

\subsubsection{Descritor Gestão da Informação}

Na UFC, observamos o descritor Gestão da Informação foi referenciado no curso de Administração, campus Fortaleza, na disciplina de Gestão do Conhecimento, como prática de modelos de avaliação na Gestão da Informação, e na disciplina de Gestão de Sistemas de Informação, englobando as políticas e estratégias de informação. Em Biblioteconomia, Fortaleza, o curso reforça a utilização do descritor no propósito de formar profissionais com capacidade para interpretar a realidade da sociedade no que compete à gestão da informação para tomada de decisão. Em Sistemas de Informação, de Crateús e Quixadá, a aplicação do descritor está na disciplina de Gestão da Informação e dos Sistemas de Informação, destacando os conceitos de dados, informação e conhecimento, e tecnologia da informação como diferencial estratégico nas organizações. Já em Engenharia de Alimentos, Fortaleza, a menção ao assunto está na disciplina de Computação Aplicada, como reforço no material bibliográfico.

Tabelas 6. Descritor Gestão da Informação nos cursos da UFC e do IFCE

\begin{tabular}{c|c|c}
\hline Instituição & Curso & Cidade \\
\hline \multirow{4}{*}{ UFC } & Administração & Fortaleza \\
\cline { 2 - 3 } & Biblioteconomia & Fortaleza \\
\cline { 2 - 3 } & Sistemas de Informação & Crateús \\
\cline { 2 - 3 } & Sistemas de Informação & Quixadá \\
\cline { 2 - 3 } & Engenharia de Alimentos & Fortaleza \\
\hline \multirow{2}{*}{ IFCE } & Sistemas de Informação & Crato \\
\hline
\end{tabular}


Entrando nos cursos do IFCE, o descritor encontra-se somente em Sistemas de Informação, Crato, citado na disciplina de Computador e Sociedade, em sua bibliografia complementar.

\subsubsection{Descritor Gestão de Processos}

$\mathrm{Na}$ UFC, o descritor é mencionado no curso de Pedagogia, Fortaleza, entre competências que devem ser desenvolvidas, sendo essas a gestão de processos educativos. Em Sistemas de Informação, Crateús, é tratado na disciplina de Organização, Sistemas e Métodos, com a designação relativa aos conceitos e requisitos de informação para a gestão de processos de negócio. Redes de Computadores, Quixadá, traz a referência inserida no desenvolvimento de competências profissionais tecnológicas, gerais e específicas, para a gestão de processos e a produção de bens e serviços. No curso de Sistemas e Mídias Digitais, Fortaleza, está na atuação profissional, tornando-se ativo na produção multimídia, direção de arte e gestão de processos.

O descritor Gestão de Processo, no IFCE, tem sua menção no curso de Ciências Biológicas, Acaraú, na disciplina de Empreendedorismo, por meio dos objetivos específicos desta, ressaltando os modelos de gestão de processos inovadores. Em Tecnologia em Construção de Edifícios, Juazeiro do Norte, as Práticas Pedagógicas definem o desenvolvimento de funções e atividades referentes à gestão de processos de produção de bens e serviços, trazendo aptidões para a pesquisa tecnológica e para a disseminação de conhecimentos tecnológicos. O curso de Saneamento Ambiental, em Sobral, define que o perfil profissional seguirá a tendência de mercado, podendo atuar na gestão de processos de recuperação de áreas degradadas.

Tabelas 7. Descritor Gestão de Processos nos cursos da UFC e do IFCE

\begin{tabular}{c|c|c}
\hline Instituição & Curso & Cidade \\
\hline \multirow{4}{*}{ UFC } & Pedagogia & Fortaleza \\
\cline { 2 - 3 } & Sistemas de Informação & Crateús \\
\cline { 2 - 3 } & Redes de Computadores & Quixadá \\
\cline { 2 - 3 } & Sistemas e Mídias digitais & Fortaleza \\
\hline \multirow{4}{*}{ IFCE } & Ciências Biológicas & Acaraú \\
\cline { 2 - 3 } & Tecnologia em Construção de Edifícios & Juazeiro do Norte \\
\cline { 2 - 3 } & Saneamento Ambiental & Sobral \\
\hline
\end{tabular}

\subsubsection{Descritor Gestão de Sistemas}

Na UFC, o descritor Gestão de Sistemas está presente no curso de Administração, Fortaleza, ocorre na disciplina de Gestão de Sistemas de Informação, fazendo-se presente também no curso de Ciências Contábeis, atribuindo competências a serem desenvolvidas na área de gestão da informação, englobando as políticas e estratégias de informação. Em Pedagogia, Fortaleza, a menção é estabelecida no ponto que trata dos objetivos do curso. $\mathrm{O}$ assunto é ressaltado na formação de conhecimentos referentes à gestão de sistemas educativo-escolares. Por fim, nesse grupo da UFC, encontra-se o 
descritor no curso de Sistemas de Informação, de Crateús e Quixadá, com sua referência presente nas áreas de atuação, ressaltando que o profissional pode atuar na gestão de Sistemas de Informação, como gerente de sistemas de informação, consultor/auditor em gestão de sistemas de informação.

No IFCE o descritor é referenciado no curso de Química, campus Aracati, na definição profissional, relacionado a gestão de sistemas de educação. No curso de Gestão de Turismo, Canindé, a associação ao descritor está na disciplina de Gestão de Sistemas de Informação, com conceitos, técnicas e ferramentas de gestão da tecnologia da informação na administração de um negócio. Em Sistemas de Informação, Crato, a presença está na relação dos objetivos e perfil dos egressos, habilitando-os a coordenar equipes de desenvolvimento e gestão de sistemas de informação. Já em Redes de Computadores, Jaguaribe, a presença está na disciplina de Gestão de Tecnologia da Informação, que propõe a gestão de sistemas: níveis de execução e serviços.

Tabelas 8. Descritor Gestão de Sistemas nos cursos da UFC e do IFCE

\begin{tabular}{c|c|c}
\hline Instituição & Curso & Cidade \\
\hline \multirow{4}{*}{ UFC } & Administração & Fortaleza \\
\cline { 2 - 3 } & Ciências Contábeis & Fortaleza \\
\cline { 2 - 3 } & Pedagogia & Fortaleza \\
\cline { 2 - 3 } & Sistemas de Informação & Crateús \\
\cline { 2 - 3 } & Sistemas de Informação & Quixadá \\
\hline \multirow{4}{*}{ IFCE } & Química & Aracati \\
\cline { 2 - 3 } & Gestão de Turismo & Canindé \\
\cline { 2 - 3 } & Sistemas de Informação & Crato \\
\cline { 2 - 3 } & Redes de Computadores & Jaguaribe \\
\hline
\end{tabular}

\subsection{Tema IV - Segurança da Informação (Descritor Segurança da Informação)}

Nos cursos da UFC, o descritor Segurança da Informação se encontra em Engenharia da Computação, Fortaleza, em uma disciplina optativa de Auditoria e Segurança da Informação. Em Redes de Computadores, Quixadá, está nas áreas de atuação profissional, e na disciplina de Sistemas Operacionais de Redes, Segurança e Serviços, e na disciplina de Segurança da Informação.

Nos cursos do IFCE, a caracterização do descritor está presente no curso de Ciências da Computação, Aracati, na disciplina optativa de Segurança da Informação, entre os princípios de segurança da informação. Em Redes de Computadores, Canindé, a única informação relativa ao descritor foi a oferta da disciplina de Gestão de Segurança da Informação. Já em Jaguaribe, no curso de Redes de Computadores, o tema se relaciona ao escopo do curso, definido nas áreas de atuação profissional como Analista de Segurança da Informação. Também é mencionado na disciplina de Segurança de Redes, como métricas de aprendizagem e conceitos sobre segurança da informação. No curso de Tecnologia em Telemática, Tauá, observa-se o resultado na disciplina de Segurança da Informação, de forma parecida à encontrada em Ciências da Computação, 
VI Congresso Brasileiro de Informática na Educação (CBIE 2017)

Anais do XXIII Workshop de Informática na Escola (WIE 2017)

Aracati, mas se diferenciando nas partes de, aspectos tecnológicos da segurança da informação e bases para desenvolvimento de planos em segurança da informação.

Tabelas 9. Descritores do Tema IV nos cursos da UFC e do IFCE

\begin{tabular}{c|c|c}
\hline Instituição & Curso & Cidade \\
\hline \multirow{4}{*}{ UFC } & Engenharia da Computação & Fortaleza \\
\cline { 2 - 3 } & Redes de Computadores & Quixadá \\
\hline \multirow{4}{*}{ IFCE } & Ciências da Computação & Aracati \\
\cline { 2 - 3 } & Redes de Computadores & Canindé \\
\cline { 2 - 3 } & Redes de Computadores & Jaguaribe \\
\cline { 2 - 3 } & Tecnologia em Telemática & Tauá \\
\hline
\end{tabular}

\section{Análise dos Resultados}

Ao longo do Tema I, Infraestruturas Críticas, repara-se uma limitada referência nos PPC, com o processo de busca retornando apenas resposta em umas das variações, e estando ele direcionado a uma subárea, os sistemas críticos. Como efeito direto dessa perspectiva, foram geradas consequências ao longo dos demais resultados, evidenciando-se que, diretamente, o tema central não é mencionado nos PPC de nenhumas das instituições, tornando-se este um elemento atípico e somente existente indiretamente, em assuntos relacionados ao contexto.

No Tema II, Gestão de Riscos, observa-se que, nem sempre, a citação do descritor no PPC reflete que o assunto está inserido no contexto da pesquisa. Nota-se, também, que os cursos em que essa temática está inserida estão voltados para graduações que lidam com riscos que podem acarretar prejuízos de nível financeiro, social e humano. Estão dispostos em ementas que visam conscientizar e gerar reflexão do futuro profissional na gestão de seu trabalho.

O Tema III compreendeu nos PPC processos voltados a formar profissionais competentes nas áreas de conhecimento tecnológico, independente de o curso ter a tecnologia como atividade principal. Na conjuntura do descritor Gestão da Informação, obteve-se uma proporção bastante significativa na UFC quanto à abordagem, porém, no IFCE, teve-se uma baixa atribuição. Os demais descritores, Gestão de Processos e Gestão de Sistemas, encontram-se estabelecidos de maneiras mais específicas, inseridos em materiais que oportunizam a integração entre recursos específicos de cada curso.

No último assunto, o Tema IV, apenas se observou, mais uma vez, que a Segurança da Informação na denotação das IC segue mecanismos parciais, e não está contextualizada a temática em nenhum dos PPC. Os descritores Proteção de Ativos e Segurança Cibernética possuem uma taxa de identificação bastante significativa com o tema central, porém não é tratada em nenhum PPC das duas instituições.

\section{Considerações Finais}

Como reflexão do estudo, chegou-se ao registro de que as IC devem ser abordadas de forma ampla e incisiva, durante o processo de graduação. A capacitação do material 
humano promoverá crescimento tecnológico e analítico, resultando em segurança para a sociedade e para os bens, diretamente, ligados às IC.

Na pesquisa documental, encontram-se irregularidades nas instituições públicas, posto que algumas não têm ainda público os PPC de seus cursos. Analisando-se os projetos, foram encontrados pontos que abordam subáreas de IC, e isso é uma demonstração de que os respectivos cursos reconhecem e aplicam no currículo alguns conteúdos da área. Mas, ao se verificar que, dos cursos de Tecnologia e Ciência, apenas os relacionados a Computação abordam algum tipo de conteúdo relacionado a IC, isso mostra uma grave falha na formação. Por exemplo, se biólogos que, durante sua vida acadêmica, não tiveram contato com a subárea de IC, Proteção de Ativos, e, hoje, trabalham em um laboratório, com um sequenciador de DNA, pela falta de maturidade no assunto, correm o risco de danificar o equipamento e apresentar prejuízos na escala de milhões caso não sejam eficientes.

Em relação a trabalhos futuros, há o entendimento de que os cursos de graduação estão cada vez mais breves (os cursos de tecnólogos são um exemplo), com a justificativa de colocar o profissional no mercado o quanto antes. Resta a pergunta sobre onde o egresso aprenderá esse conteúdo depois da graduação. A afirmação usual de que "o mercado especializará seu profissional" precisa ser melhor especificada. Nesse ponto, caberão pesquisas futuras para verificação e discussão.

Por fim, ressalta-se a relevância deste artigo para a construção do conhecimento: com base na carência encontrada, poder-se-á promover a continuidade da pesquisa, visando à criação de módulos de saberes que possam ser empregados nos cursos de graduação, reforçando as IC nesse processo.

\section{Referências}

Canongia, C., Mandarino Jr, R., \& Gonçalves Jr, A. (2010). Guia de Referência para a Segurança das Infraestruturas Críticas da Informação-Versão 01. Brasília, DSIC/SE/GSI/PR, Brasil, 151.

Hosang, A. (2011). Política Nacional de Segurança Cibernética: uma necessidade para o Brasil. Escola Superior De Guerra, Rio De Janeiro.

Lewis, T. G. (2014). Critical infrastructure protection in homeland security: defending a networked nation. John Wiley \& Sons.

Matos, E.S.; Da Silva, G. F. B. (2012). Currículo de licenciatura em computação: uma reflexão sobre perfil de formação à luz dos referenciais curriculares da SBC. In Anais do XX Workshop de Educação em Computação.

Mishra, S., Raj, R. K., Romanowski, C. J., Schneider, J., \& Critelli, A. (2015). On building cybersecurity expertise in critical infrastructure protection. In Technologies for Homeland Security (HST), 2015 IEEE International Symposium on (pp. 1-6). IEEE.

Mishra, S., Romanowski, C. J., Raj, R. K., Howles, T., \& Schneider, J. (2013). A curricular framework for critical infrastructure protection education for engineering, technology and computing majors. In Frontiers in Education Conference, 2013 IEEE (pp. 1779-1781). IEEE. 